\title{
Critical Factors and Multisite Implementation of ERP: A Case Study in the UAE
}

\author{
Wenshin Chen
}

Abu Dhabi University, Abu Dhabi, UAE

\begin{abstract}
Despite extensive studies in the ERP literature, little empirical understanding has been reached in relation to ERP implementation experiences in developing markets, particularly in the UAE context. Derived from the notions of critical successful factors and multisite implementation, two of widely researched areas in ERP studies, this paper thus seeks to provide practical insights about organizations' ERP implementation experiences in the UAE setting. More specifically, it describes and contrasts critical factors and multisite implementation experiences in two case organizations situated in the UAE. These case organizations, one being categorized as a global company and the other local, provide interesting comparison of ERP implementation because of their complementary organizational structure and business strategies. In contrast to traditional ERP frameworks' suggestions, these case organizations' experiences reveal that contemporary ERP implementations might be more complex than previously expected since none of these case organizations' ERP experiences follows suggestions made by frameworks based. Further discussion about how to better understand and examine maturing ERP technology in an increasingly globalized business environment such as the UAE is provided.
\end{abstract}

Keywords: ERP, UAE, Critical Factors, Multisite Implementation.

\section{Introduction}

Although ERP (enterprise resource planning) has been widely studied in the IT (information technology) literature, empirical understanding about ERP implementation in developing markets is relatively limited. While thousands of articles can be easily found in electronic database search, few specifically target developing economies. For example, an EBSCOhost database search with "ERP" as title generated 7742 articles in March 2012 including 3283 scholarly (peer reviewed) journal articles. However, only 9 peer reviewed journal articles were found after adding "developing countries," "developing markets," or "developing economies" in all fields as search criteria. This search experience shows that, despite the extensive existing literature, ERP research can still find a niche in developing economies. More specifically, existing studies examining ERP implementation in developing countries rarely focus on comparisons between local and global organizations. While it is important to understand different ERP implementation experiences in developing markets in general and in the UAE (United Arab Emirates) context in particular due to their growing contribution to the world's economy and to the IT industry (Chen, 2009; Chen, 2011), it is particularly informative to compare and contrast ERP experiences between local and global organizations situated in the same developing market, such as the UAE (Chen, 2012).

As Markus, Tanis and Van Fenema (2000a) argue, organizations situated in different geographic locations with various organizational structures are likely to adopt different business strategies, software configuration, technology 
platform and/or practical execution for their ERP implementations. In other words, a local company completely based in the UAE context and a global company with multiple businesses or divisions in different countries will have different business and technology strategies for their ERP implementations. Similarly, other researchers have suggested certain critical success factors for ERP implementation since the early 2000s. Commonly recommended ones include top management support, strategic objectives, implementation team, education and training and business process reengineering (BPR) (Chen, 2012). The first two factors are mostly related to an organization's strategic perspectives and can vary from company to company (Miles and Snow, 1986; Porter, 1987), particularly from a local to a global one (Chen, 2012). The last three factors focus primarily on operational levels and certainly differ in organizations, teams, or business environment, particularly in relation to BPR (McAdam, 2002).

Moreover, the process theory suggested that ERP implementation is not just about factors involved but more importantly about the process through which it evolves and interacting effects among factors involved (Koh, Soh and Markus, 2000; Tarafdar and Roy, 2003). As such, understanding context factors for ERP implementation is imperative because these factors might be unique to organizational culture or business processes that would significantly influence how ERP projects are carried out (Dezdar and Ainin, 2011; Kouki, Poulin and Pellerin, 2010). Recent empirical literature has thus witnessed increasing attention paid to unique local contexts such as China (Chien et al., 2007; Martinsons, 2004; Reimers, 2003), India (Poti and Kamalanabhan, 2009; Tarafdar and Roy, 2003) and the Middle East (Al-Turki, 2011; Amid, Moalagh and Zare Ravasan, 2012; Baki and Çakar, 2005). However, no empirical study has been found in the UAE context or based on contrasting theoretical frameworks. To shorten this knowledge gap, this study thus seeks to extend the abovementioned research stream to the
UAE context and apply contrasting theoretical guidelines to help illustrate how ERP projects are implemented in the UAE setting. Specific research questions inquire, "What are ERP implementation experiences in the UAE context?" "What are the differences of ERP implementation between local and global companies situated in the UAE?"

\section{ERP Framework}

Since the late 1990s and early 2000s, numerous frameworks for ERP implementations have been widely discussed (Parr and Shanks, 2000). At the earlier stage, much discussion focused on exploratory experiences (Markus et al., 2000) or specific factors such as the role of CIO (Willcocks and Sykes, 2000) and cultural fit (Soh, Kien and Tay-Yap, 2000). Another stream of empirical studies examined the process, instead of factors, through which ERP implementation evolve (Tarafdar and Roy, 2003). It was suggested that ERP implementation could evolve into chartering, project, shakedown, and onward and upward phases with each phase facing certain common errors that need to be addressed accordingly (Koh et al., 2000). Perhaps due to its emerging nature at that time, research endeavor was much concerned with how to ensure ERP implementation's success (Scheer and Habermann, 2000).

This enormous concern about ERP success has led to extensive discussion on the two major frameworks that will be discussed in the following sections: multisite ERP implementation (Markus, Tanis and van Fenema, 2000) and critical success factors (CSFs) (Akkermans and van Helden, 2002; Nah, Zuckweiler and Lau, 2003); the former provides a comprehensive overview for complex ERP implementation and the latter suggests various factors that organizations need to seriously consider while managing ERP projects. These two frameworks are chosen as the theoretical foundation for this study because they are most relevant to the research questions addressed earlier. More specifically, most ERP implementations in contemporary globalized economy, whether they are 
localized or on a global scale, are complex and difficult (Markus et al., 2000). The multisite implementation framework can provide an integrative understanding of local vs. global ERP implementations (i.e. research question two). Critical success factors, on the other hand, suggest some of most comprehensive issues that revolve around ERP implementations (Olson, 2004). These factors have constituted arguably the most widely cited guideline in the literature that ERP implementation in the UAE context cannot afford to overlook (i.e. research question one).

\section{Multisite Implementation Framework}

The significance of multisite implementation framework is that it articulates different interdependent layers of ERP implementations. In other words, the choices made in one layer, particularly on the upper layers, can affect choices or resources available in another. Therefore, it is highly recommended that the planning of ERP implementations starts at the upper level (i.e. strategic level) and then progresses into the technical levels accordingly (Markus et al., 2000). Four layers of interdependent factors discussed in the multisite implementation framework are business strategy, software configuration, technology platform, and practical execution.

In planning business strategy, Markus, Tanis and van Fenama (2000) suggest five options ranging from total decentralization to total centralization.

- Total Local Autonomy: this complete decentralization strategy allows local business units full control of their own decision making and ERP implementation. It values local culture and contextual factors but could face difficulty in ERP integration.

- Centralized Financial Control: this largely decentralization strategy allows local business units most decision making and ERP implementation except for financial modules. It can be effective when various units or branches conduct different businesses but are integrated into one 'best-of-breed' financial system.

- Centralized Operations Coordination: this strategy allows most localized operations, but headquarters are involved in operations concerning global supply chain. It can be effective when common advantages of procurement or logistics management are realized. Headquarters' involvement in ERP project implementation, however, should be highly expected.

- Network Operations Coordination: this strategy allows local units network access to various operations information and thus provides a lateral coordination. It is most effective when units themselves transact with one another as well as with external customers.

- Total Centralization: this strategy is a typical top-down approach that will centralize decision making at headquarters and present a common corporate image to the business world.

The second layer of implementation factors discussed in multisite framework is about software configuration. Four approaches are suggested revolving around financial and operational modules.

\section{Multiple Financial/Multiple} Operations: this approach is most appropriate for organizations with multiple facilities in multiple countries and generally compatible with the total decentralization strategy abovementioned.

- Multiple Financial/Single Operation: this approach is unique for organizations with a single production and operations facility but with multiple sales divisions.

Single Financial/Multiple Operations: this type of configuration can be considered for organizations with complex business processes across units but with centralized financial/legal purpose. 
- Single Financial/Single Operation: this type of configuration is most suitable for simple, centralized organizations. Complex organizations can also consider this approach if they have centralized financial purpose and their units have similar business processes.

The third layer of implementation factors addresses issues of technology platform. It is mainly centered on distributed or centralized architectures.

- Distributed Architecture: despite greater challenge in implementation, this type of architecture is more suitable for organizations with multiple units or facilities that demand high performance in data access and local management autonomy. It is generally more suitable for the total decentralization strategy and multiple software configurations.

- Centralized Architecture: this type of technology platform is often easier to implement and manage. However, for organizations with geographically dispersed units or facilities, this type of architecture could be more challenging due to various data access and network performance issues.

The last layer of implementation factors involved is practical execution. Two major approaches, big bang and phased rollout, are discussed.

- Big Bang: this radical and risky approach is to discontinue old systems and replace them with new systems all at once. As its name implies, this type of deployment might often cause major disruption in organizational operations. However, empirical studies have found that big bang deployment is more commonly practiced than phased rollout among organizations surveyed in the US and Sweden (Olson, 2004).

- Phased Rollout: this incremental and smooth approach is to deploy ERP systems step by step. It often provides adjustment periods for new systems to replace the old ones and allow users or different units/branches to be accustomed to the new ones. Long term commitment and dedication is often required for this approach.

In relation to this study's research questions, multisite implementation framework would likely suggest that local companies' ERP implementations will be substantially different from those of global corporations due to their contrasting enterprise purposes, geographical sites, business processes and operations, and available resources. Perhaps a global company will be more likely to adopt a decentralized approach, either from the strategic level or from the technical levels, due to the complexity of their business operations and ERP implementations.

\section{CSFs Guideline}

While multisite implementation framework provides four layers of ERP considerations, particularly for complex implementations, critical success factors could serve a comprehensive guideline for all types of ERP projects. It is arguably one of the most widely studied areas in the ERP literature. While some studies focus on organizational or managerial issues, others tend to discuss technical details or project factors. For example, $\mathrm{Nah}$ et al. (2003) originally discussed ten critical success factors from their literature review. Their empirical study on CIO's (Chief Information Officer) perceptions suggested that five most significant factors are top management support, project champion, project team, project management, and change management. None of these factors is related to specific technical factors. In contrast, other researches consider certain technical issues, such as data accuracy (Olson, 2004) or package selection (Akkermans and van Helden, 2002) to be significant. However, as Akkermans \& van Helden (2002) suggests, many critical success factors might be interrelated to one another which might shape ERP implementation into a more complex and difficult process than organizations desire.

To conclude, CSFs guideline seemingly suggests that ERP implementation involve 
many organizational, managerial, and project factors that are mostly beyond technical measures. More specifically, the only two factors that are commonly agreed upon, among five CSFs articles reviewed, are top management support and implementation team. Other factors that are considered by at least three articles are strategic objectives, project management implementation, education and training, and BPR (business process reengineering).

- Strategic Objectives: this refers to an organization's clear understanding of its strategic objectives and goals. A clear vision of such objectives can better guide the directions of ERP implementation.

- Top Management Support: this factor is arguably the most widely cited success factor for not just ERP implementation but also other information systems projects. It appears to drive other managerial or technical elements to perform more properly.

- Project

Management Implementation: this project factor involves how an organization manages its ERP projects. Common project management factors such as time, budget and quality should then be taken into consideration as well.

- Implementation Team: the team involved in ERP project could consist of business and IT representatives. How team members collaborate will likely determine how the project is carried out.

- Education and Training: this factor might concern mostly the users. For an ERP project to succeed, users will need to understand its benefits and new functions. Better education and training can facilitate users' further cooperation and complete utilization of ERP systems in the end.

- BPR: this factor stresses how to renovate business processes so that a better integration of ERP system with existing organizational functions can be achieved.
In relation to this study's research questions, this CSFs guideline would most likely suggest that ERP implementation experiences in the UAE will face more organizational, managerial and project factors than technical issues. To better implement and manage ERP projects, organizations will need to better deal with these issues successfully. Furthermore, factors that are significant to a local company might differ from those of a global company since their strategic objectives, top management mentality, project resources, implementation experiences and local business processes all vary. However, since factors summarized above are not drawn from studies conducted in unique local economies such as the UAE, the aforementioned argument remains to be examined.

\section{Methodology}

Based on these two frameworks, this study embarked on a qualitative research methodology with a specific category of descriptive case study. Case study approach is commonly recognized as the most widely adopted qualitative methodology (Chen and Hirschheim, 2004). It is most suitable for investigating complex issues situated in clearly defined boundaries (Eisenhardt, 1989). While various suggestions have been made, three primary case study researches can be categorized: exploratory, descriptive and explanatory (Yin, 1994). According to Yin (1994), exploratory case study is most appropriate for investigating emerging issues that lack existing understanding in the literature. Explanatory case study, in contrast, seeks to clarify complex issues in participant organizations, provide implications to their work practices, and perhaps enhance theoretical understanding of the phenomenon or cases investigated. Descriptive case study is mostly fitting for providing case descriptions as the way participant organizations are and for deriving practical lessons or insights from those case descriptions. Descriptive case study is considered appropriate for the study because it suits research purpose and research context where in-depth 
explanation appears infeasible due to insufficient data access. Information gathered for case description below is derived from public sources while ERR experiences descried in the section that follows are based on personal contacts and interviews in participant organizations.

\section{Case Description}

Two case companies chosen for this report are given pseudonyms: Libra Gas and Pisces Petroleum. The reason for selecting these two cases is mostly because they are situated in the same business context, the oil and gas industry, which constitutes a major economy in the country and, more interestingly, their contrasting operations, one local and one global, which serve the research purpose.

Libra Gas is a local company specializing in certain productions in the oil and gas industry. It is owned by several major oil companies locally and globally. While its headquarter is located in the metropolitan area where this research project took place, there are several production facilities in the country. In relation to the multisite implementation framework, Libra Gas's situation is a typical centralized ERP case. Pisces Petroleum, on the other hand, has operations all over the world involving all aspects of oil and gas businesses. It is a publicly traded company headquartered in one of the major metropolitans in the world. The subsidiary in the metropolitan area where the research project took place has been present in the UAE since the early 1900s. It currently holds considerable amount of interests in local oil and gas companies. Based on the multisite implementation framework, Pisces Petroleum is a typical decentralized ERP case due to its multifaceted, complex operations in many countries.

\section{ERP Experiences}

ERP project in Libra Gas involved three phases over a period of 18 months. The planning phase took 3 months to prepare. The second phase mostly centered on vendor evaluation and contract negotiation while the third phase, the actual implementation process, was carried out over 11 months. Stakeholders involved in ERP project included IT steering committee, ERP steering committee, EPR project manager who supervised and coordinated project resources which primarily consisted of business team, IT team and ERP consultants and vendors. Despite its typical case of complete centralized ERP project, Libra Gas chose a decentralized strategy that headquarters would only control its finances and allow separate ERP modules for local units and facilities. Subsequently, its software configuration was based on single financial/multiple operations approach and technology platform was largely derived from distributed architecture, except for financial module that was standardized and controlled by its parent company. Finally, Libra Gas chose the phased rollout approach, more specifically phased rollout by modules, for its ERP execution.

Most critical factors that affected Libra Gas's ERP implementation were top management support, learning from external resources, user involvement, strategic objectives, performance measures and implementation team.

Top Management Support: Libra Gas's top management provided necessary resources to enable ERP implementation from the outset.

Learning from External Resources: prior to ERP implementation, Libra Gas consulted two external companies in the same industry that have ERP experiences. Lessons learned from those two companies' issues and mistakes facilitated Libra Gas's ERP project considerably.

User Involvement: ERP project team made necessary attempt to involve users in ERP implementation process. This included consulting with a business team in the process and educating users with functions and benefits of ERP systems at earlier stages. However, this factor might not be managed appropriately because it was found that 
user resistance became a major issue after ERP implementation.

- Strategic Objectives: prior to implementation, Libra Gas has developed clear strategic objectives of ERP project for internal operations and competitive reasoning.

- Performance Measures: this factor was managed by weekly reports and ERP newsletters distributed to the entire company. Monthly meetings were also held to ensure ERP project meet its requirement and expectation.

- Implementation Team: Libra Gas's own specialists and one of its major vendors' staff created an atmosphere that encouraged collaborative interaction among all stakeholders which in turn helped smooth out the implementation phase.

In contrast, ERP implementation in Pisces Petroleum involved four phases over a period of approximately 8 months. The first phase involved only one month of project preparation and the second phase immediately followed with two and half months of business blueprint development. The following two months involved project realization, while the last phase was the final preparation which was completed in slightly less than two months. The ERP project was mostly supervised by two project managers who coordinated and oversaw five participating teams: IT and data, supply, finance, region support, and plant maintenance. Despite its typical decentralized ERP case, Pisces Petroleum embarked on a total centralization strategy. Interestingly, its software configuration did not follow single finance/single operation approach that would be most common for a total centralization strategy. Instead, Pisces Petroleum chose a multiple finance/single operation approach for its software configuration. What further complicated its implementation process was its dual architectures for technology platform. More specifically, Pisces Petroleum provided both centralized databases at its headquarter and at the same time distributed servers over many regions. Lastly, its practical execution revolved around the phased rollout approach with incremental implementation by sites and modules.

Five most critical factors that affected Pisces Petroleum's ERP implementation in the UAE were top management support, extensive education and training, balanced implementation team, clear understanding of strategic objectives, and commitment to change.

- Top Management Support: the strategic planning for Pisces Petroleum's ERP project specifically required top management's commitment from the outset. All departmental chairpersons were involved in ERP planning at all stages.

Education and Training: Pisces Petroleum provided formal forums, such as meetings and training sessions, and indirect communications, such as ERP newsletters to familiarize users with new ERP systems. Both computerbased and in house trainings were available for users.

- Implementation Team: Pisces Petroleum's ERP project included a specifically dedicated IT and data team, and four different business teams that provided various business perspectives to help integrate ERP systems into the business processes.

Strategic Objectives: as mentioned previously, Pisces Petroleum's first two implementation phases primarily involved developing a better understanding of its business strategy and project purpose. Due to its centralization approach, most objectives and goals were also specified by the headquarters.

- Commitment to Change: Pisces Petroleum was aware of psychological effects that might cause users' resistance to change. As such, it initiated several workshops in different 
implementation phases to provide support to users.

\section{Discussion}

Concerning the first research question, 'what are ERP implementation experiences in the UAE context?', two cases described above demonstrated that despite the vast body of knowledge in the existing ERP literature, ERP experiences in the UAE did not appear as what various frameworks might suggest. For the multisite implementation framework, ERP implementation in the UAE evidently developed into the opposite direction. A typical centralization case such as Libra Gas would eventually adopt a decentralization approach while a seemingly predictable decentralization case such as Pisces Petroleum chose a total centralization approach. This finding suggests that ERP implementation might be more complex and unpredictable as previously recommended. Further investigation will be needed to understand these complex issues involved.

With respect to the second research question, 'what are the differences of ERP implementation between local and global companies situated in the UAE?', the contrasting cases of Libra Gas and Pisces Petroleum, one local and the other global, sparkled further research interests. It was perhaps expected that there existed substantial differences in ERP implementation experiences between a local and a global company. What was unexpected is how they differed. More specifically, a local company such as Libra Gas demonstrated certain ERP experiences that were traditionally expected in a global corporation, while a global company such as Pisces Petroleum contrastingly exhibited ERP characteristics that were mostly expected in a local company. Based on CSFs guideline, these two case ERP experiences also resulted in different focuses. Only one common factor, top management support, was cited by both companies as the utmost priority. Two other factors, upon which both companies agreed, balanced implementation team and clear understanding of strategic objectives, were ranked differently. Most significantly, a local company such as Libra Gas seems to be concerned more about learning from external resources and measuring project performance accurately, whereas a global company such as Pisces Petroleum is mostly concerned about managerial and organizational factors that were traditionally expected.

One possible explanation for such unexpected results might be due to these companies' prior ERP implementation experiences. For Pisces Petroleum, since it has previously implemented ERP in various branches and global market, it might be easier to model after their prior successful implementation processes and adopt a centralized approach that could simplify and facilitate the implementation process. On the contrary, Libra Gas has no prior ERP implementation experience. Its top management thus might tend to rely on external consultants and implementation team involving those who have better technical expertise. It could help explain why they focus critical factors on learning from external resources and measuring their performance. This possible mentality might also lead to its eventual decentralized approach that allows different stakeholders or branches involved to adopt a more fitting module for their own operational preferences.

\section{Conclusion}

Although only two cases were reported and contrasted, this study has provided new insights for future ERP research. First, traditional ERP frameworks might provide a comprehensive guideline for empirical researches. They cannot universally comprehend how ERP projects evolve in local contexts such as the UAE. While recent ERP researches have suggested the significance of contextual factors, little prior understanding of how local and global companies in emerging markets such as the UAE would demonstrate different ERP strategic choices in a direction that is completely unexpected. Is it due to the headquarters' involvement and control or IT management's strategic planning? Or is it because the mature ERP 
market in the global landscape has enabled companies, local or global, to see implementation approach differently from traditional frameworks' perspectives?

Second, critical factors involved, except for top management support, appear in different levels of priority in a local company from a global one. A local company such as Libra Gas seems to be more interested in learning from external resources so that unnecessary mistakes can be avoided, while a global company such as Pisces Petroleum appears rather comfortable in settling into a routine where most critical factors are largely expected. Is it because a global company possesses more available resources so that it would expectedly follow its prior experiences? Or it is due to specific organizational context where a local company which was largely owned by other major corporations might need to care about better survival measurement? Further research investigation is apparently needed to provide a deeper understanding of how these issues evolve.

\section{Acknowledgement}

An earlier version of the paper was presented at the $18^{\text {th }}$ IBIMA Conference, Istanbul, May 2012.

\section{References}

Akkermans, H. \& van Helden, K. (2002). "Vicious and Virtuous Cycles in ERP Implementation: A Case Study of Interrelations between Critical Success Factors," European Journal of Information Systems, 11, 35-46.

Al-Turki, U. M. (2011). "An Exploratory Study of ERP Implementation in Saudi Arabia," Production Planning \& Control, 22 (4), 403-413.

Amid, A., Moalagh, M. \& Ravasan, A. Z. (2012). "Identification and Classification of ERP Critical Failure Factors in Iranian Industries," Information Systems, 37 (3), 227-237.
Baki, B. \& Çakar, K. (2005). "Determining the ERP Package-Selecting Criteria: The Case of Turkish Manufacturing Companies," Business Process Management Journal, 11(1), 75-86.

Chen, W. (2009). "E-Services and Tourism: A Case Study of UAE Hotel Industry," The Ninth International Conference on Electronic Business (ICEB), November 30December 4, Macau, China.

Chen, W. (2011). "Cloud Computing in the UAE Context: An Institutional Perspective," International Conference on Information Resource Management (Conf-IRM), June 12-14, Seoul, South Korea.

Chen, W. (2012). "From Local to Global: ERP Implementation Experiences in the UAE" the 18th IBIMA (International Business Information Management Association) Conference, May 9-10, Istanbul, Turkey.

Chen, W. \& Hirschheim, R. (2004). "A Paradigmatic and Methodological Examination of Information Systems Research from 1991 to 2001," Information Systems Journal, 14(3), 197-235.

Chien, S.- W., Hu, C., Reimers, K. \& Lin, J.S. (2007). "The Influence of Centrifugal and Centripetal Forces on ERP Project Success in Small and Medium-Sized Enterprises in China and Taiwan," International Journal of Production Economics, 107(2), 380-396.

Dezdar, S. \& Ainin, S. (2011). "The Influence of Organizational Factors on Successful ERP Implementation," Management Decision, 49(6), 911-926.

Eisenhardt, K. M. (1989). "Building Theories from Case Study Research," Academy of Management Review, 14(4), 532-550.

Koh, C., Soh, C. \& Markus, M. L. (2000). 'A Process Theory Approach to Analyzing ERP Implementation and Impacts: The Case of Revel Asia,' Journal of Information Technology Cases \& Applications, 2(1), 4-23. 
Kouki, R., Poulin, D. \& Pellerin, R. (2010). 'The Impact of Contextual Factors on ERP Assimilation: Exploratory Findings from a Developed and a Developing Country,' Journal of Global Information Technology Management, 13(1), 28-55.

Markus, M. L., Axline, S., Petrie, D. \& Tanis, C. (2000). "Learning from Adopters' Experiences with ERP: Problems Encountered and Success Achieved," Journal of Information Technology (Routledge, Ltd.), 15(4), 245-265.

Markus, M. L., Tanis, C. \& van Fenema, P. C. (2000). "Multisite ERP Implementations," Communications of the $A C M, 43(4), 42-46$.

Martinsons, M. G. (2004). "ERP in China: One Package, Two Profiles," Communications of the ACM, 47(7), 65-68.

McAdam, R. (2002). "Large Scale Innovation - Reengineering Methodology in SMEs," International Small Business Journal, 20(1), 33-52.

Miles, R. E. \& Snow, C. C. (1986). "Organizations: New Concepts for New Forms," California Management Review, 28(3), 62-73.

Nah, F. F.- H., Zuckweiler, K. M. \& Lau, J. L.S. (2003). "ERP Implementation: Chief Information Officers' Perceptions of Critical Success Factors," International Journal of Human-Computer Interaction, 16(1), 5-22.

Olson, D. L. (2004). Managerial Issues of Enterprise Resource Planning Systems, McGraw Hill New York.

Parr, A. \& Shanks, G. (2000). "A Model of ERP Project Implementation," Journal of Information Technology, 15, 289-303.

Porter, M. E. (1987). "From Competitive Advantage to Corporate Strategy," Harvard Business Review, 65(3), 43-59.

Poti, S. \& Kamalanabhan, T. J. (2009). "Evaluation and Importance of a PreChange Stage in an Enterprise Resource Planning (ERP) Implementation
Perspective in India: A Conceptual Framework," Journal of Transnational Management, 14(3), 241-256.

Reimers, K. (2003). "Implementing ERP Systems in China," Communications of AIS, 2003(11), 335-356.

Scheer, A.- W. \& Habermann, F. (2000). "Making ERP a Success," Communications of the ACM, 43(4), 57-61.

Soh, C., Kien, S. S. \& Tay-Yap, J. (2000). "Cultural Fits and Misfits: Is ERP a Universal Solution?," Communications of the ACM, 43(4), 47-51.

Tarafdar, M. \& Roy, R. K. (2003). "Analyzing the Adoption of Enterprise Resource Planning Systems in Indian Organizations: A Process Framework," Journal of Global Information Technology Management, 6(1), 31-51.

Willcocks, L. P. \& Sykes, R. (2000). "The Role of the CIO and It Function in ERP," Communications of the ACM, 43(4), 32-38.

Yin, R. K. (1994). Case Study Research: Design and Methods, SAGE Publications Thousand Oaks, CA. 